\title{
Reviewer Acknowledgements
}

Journal of Agricultural Science wishes to acknowledge the following individuals for their assistance with peer review of manuscripts for this issue. Their help and contributions in maintaining the quality of the journal are greatly appreciated.

Journal of Agricultural Science is recruiting reviewers for the journal. If you are interested in becoming a reviewer, we welcome you to join us. Please find the application form and details at http://jas.ccsenet.org/editor/recruitment and e-mail the completed application form to jas@ccsenet.org

\section{Reviewers for Volume 11, Number 9}

Abdelnaser A. Elzaawely, Tanta University, Egypt

Amir Raza, Nuclear Institute for Food and Agriculture, Pakistan

Andrés J. Pena-Q., Washington State University, United States of America

Augusto Loni, University of Pisa, Italy

B. Adebanji, Ekiti State University, Nigeria

Beatriz Sevilla-Moran, National Institute for Agricultural and Food Research and Technology, Spain

Carolina M. Sánchez-Sáenz, Universidad Nacional de Colombia, Colombia

Chunlin Yang, Ohio State University, United States of America

Clerison Perini, Universidade Federal de Santa Maria, Brazil

Danielle Fabíola Pereira da Silva, Universidade Federal de Goiás, Brazil

Darwin Pangaribuan, Lampung University, Indonesia

Fábio Gelape Faleiro, Empresa Brasileira de Pesquisa Agropecuária, Brazil

Funso Raphael Kutu, North-West University, South Africa

Germani Concenco, Embrapa Clima Temperado, Brazil

Hector Alonzo Gomez Gomez, National University of Agriculture, Honduras

Ismael Montero Fernandez, Federal University of Roraima, Brazil

Jean Carlo Possenti, Universidade Tecnológica Federal do Paraná, Brazil

Jéfferson de Oliveira Costa, Universidade de São Paulo, Brazil

Joao Everthon da Silva Ribeiro, Universidade Federal da Paraíba, Brazil

Kavita Sharma, Idaho State University, United States of America

Léo O. D. Marques, Federal University of Pelotas, Brazil

Lorena Figueira de Santana, University of Parana, Brazil

Luma R. L. Nunes, Federal University of Ceará, Brazil

Maria Aparecida de Matos, State University of Londrina, Brazil

Maria Itria Ibba, Washington State University, United States of America

María Serrano, Miguel Hernández University, Spain

Marlene Bezerra, Federal University of Paraíba, Brazil

Neyton Miranda, Federal Rural University of the Semiarid, Brazil

Renan Francisco Rimoldi Tavanti, São Paulo State University “Julio de Mesquita Filho”, Brazil

Slawomir Borek, Adam Mickiewicz University, Poland 
Tata Emmanuel Sunjo, University of Buea, Cameroon

Toshik Iarley da Silva, Federal University of Viçosa, Brazil

Vitor L. Nascimento, Universidade Federal do Tocantins, Brazil

Wellytton Quequeto, Instituto Federal de Educação, Ciência e Tecnologia Goiano, Brazil

Zhiqiang Cai, University of Pennsylvania, United States of America 